\title{
Squeezing the Local Oscillator Does Not Improve Signal-to-Noise Ratio in Heterodyne Laser Radar
}

\author{
Mark A. Rubin and Sumanth Kaushik \\ Lincoln Laboratory \\ Massachusetts Institute of Technology \\ 244 Wood Street \\ Lexington, Massachusetts 02420-9185 \\ \{rubin,skaushik\}@LL.mit.edu
}

\begin{abstract}
The signal-to-noise ratio for heterodyne laser radar with a coherent target-return beam and a squeezed local-oscillator beam is lower than that obtained using a coherent local oscillator, regardless of the method employed to combine the beams at the detector.

OCIS codes: $270.0270,270.6570,280.5600,040.2840$
\end{abstract}

$\square$

Squeezed light holds promise for reducing noise in optical-detection applications below the level obtainable using coherent light such as that emitted by lasers.1 However, squeezing is degraded by loss.2 In laser radar applications, the loss in the target-return beam-the beam received by the radar system after reflection from a target - is severe $!^{3}$ Therefore squeezing is not useful in laser radar, at least not when applied to the target-return beam.

This still leaves open the possibility that squeezed light could be profitably employed as the local oscillator (LO) in a heterodyne laser radar. In such a system the targetreturn beam is combined with a local-oscillator beam of a different frequency on a photosensitive detector, and the presence of a target is inferred from observation of oscillation of the detector response at the difference frequency of the two beams. ${ }^{[3}$ A heterodyne laser radar system combining the target-return and LO beams on a single detecting element has been proposed by Li et al. ${ }^{4}$ Their work has been criticized by Ralph ${ }^{5}$ on the grounds that the method they employ to combine the target-return beam and LO beam on the detector introduces sufficient noise to cancel out any improvement in signal-to-noise ratio (SNR) due to squeezing. However, one might envision employing other methods of combining the beams which do not add noise; e.g., using a Fabry-Perot etalon ${ }^{6}$ that reflects the LO frequency and transmits the targetreturn frequency.

(This approach is to be distinguished from a "balanced" heterodyne system in which the two beams are directed to two detectors using a beam splitter. Quantum

This work was sponsored by the Air Force under Air Force Contract FA8721-05-C-0002. Opinions, interpretations, conclusions, and recommendations are those of the authors and are not necessarily endorsed by the U.S. Government. noise in balanced heterodyne detection with squeezed light has been examined by Yuen and Chan ${ }^{7}$ and by Annovazzi-Lodi et al. ${ }^{[8}$ We would not expect such a system to benefit from squeezing, since both beams must pass through the beam splitter and thus suffer squeezingdestroying loss. In fact, a calculation of the SNR in the balanced case gives the same result as that obtained below in the present case, eq. 225), except for the change of $\cos ^{2}$ to $\sin ^{2}$ due to the $\pi / 2$ phase change of the reflected light at the beam splitter. The detection of a Doppler beat signal using a squeezed LO, as proposed by $\mathrm{Li}$ et $a l ., \frac{9}{1}$ is also an example of a heterodyne measurement to which the considerations of the present paper apply.)

Here, we show that a heterodyne detection scheme combining a coherent target-return beam and a squeezed LO beam on a single detector will fail to improve SNR regardless of the method used to combine the beams.

For target detection using the statistic $S, 10$

$$
\mathrm{SNR}=\left(\left\langle\psi_{1}|\widehat{S}| \psi_{1}\right\rangle-\left\langle\psi_{0}|\widehat{S}| \psi_{0}\right\rangle\right)^{2} / \operatorname{Var}_{0} S
$$

where $\left\langle\psi_{1}|\widehat{S}| \psi_{1}\right\rangle$ is the mean value of $S$ in that quantum state, $\left|\psi_{1}\right\rangle$, in which the target is present, $\left\langle\psi_{0}|\widehat{S}| \psi_{0}\right\rangle$ is the mean value of $S$ when the target is absent, and $\operatorname{Var}_{0} S$ is the variance of $S$ in the target-absent condition,

$$
\operatorname{Var}_{0} S=\left\langle\psi_{0}\left|\widehat{S}^{2}\right| \psi_{0}\right\rangle-\left\langle\psi_{0}|\widehat{S}| \psi_{0}\right\rangle^{2}
$$

In choosing pure quantum states to correspond to the target-present and target-absent conditions we are assuming the absence of additional non-quantum sources of noise, e.g., thermal noise, which would have to be treated using the density operator formalism. 11

For heterodyne detection,

$$
\widehat{S}=\tau^{-1} \int_{0}^{\tau} d t \cos \left(\omega_{H} t+\theta_{H}\right) \widehat{I}(t),
$$


where $\widehat{I}(t)$ is the quantum operator corresponding to the photoelectric current produced by the detector at time $t$ and $\tau$ is the fixed time interval during which the target present/absent decision is to be made. That is, $\widehat{S}$ is the Fourier component of the photoelectric current at frequency $\omega_{H}$ and phase $\theta_{H}$. We take $\omega_{H}$ to be equal to the difference between the respective frequencies of the target-return and LO beams,

$$
\omega_{H}=\omega_{T}-\omega_{L O} .
$$

(For simplicity we will always take $\omega_{T}-\omega_{L O}>0$.) So $\widehat{S}$ corresponds to detection of the beat frequency between the LO and the target-return beam.

For suitable broadband detectors, the operator corresponding to the photoelectric current at time $t$ is $\frac{12}{}$

$$
\widehat{I}(t)=\kappa \widehat{E}^{(-)}(t) \widehat{E}^{(+)}(t)
$$

where $\kappa$ is a constant, $\widehat{E}^{(-)}(t)=\left(\widehat{E}^{(+)}(t)\right)^{\dagger}$, and $\widehat{E}^{(+)}(t)$ is the positive-frequency part of the time-dependent electric field operator at the detector,

$$
\widehat{E}^{(+)}(t)=\sum_{k} i\left(\frac{\hbar \omega_{k}}{2 \varepsilon_{0} V}\right)^{1 / 2} \widehat{a}_{k} \exp \left(-i \omega_{k} t\right) .
$$

The mode frequencies are $\omega_{k}=c k$ where the wavenumber $k$ runs over the values $k=2 \pi n / V^{1 / 3}, n=1,2, \ldots$. In writing $\widehat{E}^{(+)}(t)$ as in 6 we are assuming that the detector is only sensitive to a single direction of polarization (which is the direction in which both the LO and target-return beam will be polarized) and that the optical system is such that, for each frequency, only a single spatial mode need be considered (that mode with wave vector normal to the detector surface). The annihilation operators $\widehat{a}_{k}$ satisfy the usual commutation relations,

$$
\left[\widehat{a}_{k}, \widehat{a}_{l}\right]=\left[\widehat{a}_{k}^{\dagger}, \widehat{a}_{l}^{\dagger}\right]=0, \quad\left[\widehat{a}_{k}, \widehat{a}_{l}^{\dagger}\right]=\delta_{k l} .
$$

Using (3)-(6) we obtain, in the limit $\tau \rightarrow \infty$,

$$
\begin{gathered}
\widehat{S}=\sum_{l, k \text { s.t. }\left|\omega_{l}-\omega_{k}\right|=\omega_{H}}\left(\omega_{l} \omega_{k}\right)^{1 / 2} \\
\widehat{a}_{l}^{\dagger} \widehat{a}_{k} \exp \left(-i \varepsilon\left(\omega_{l}-\omega_{k}\right) \theta_{H}\right),
\end{gathered}
$$

where

$$
\varepsilon(x)=\operatorname{sign} \text { of } x .
$$

In the target-absent state, all modes but the LO are in the vacuum state:

$$
\left|\psi_{0}\right\rangle=|\alpha, \xi\rangle_{k_{L O}} \prod_{k \neq k_{L O}}|0\rangle_{k}
$$

Here $|\alpha, \xi\rangle_{k_{L O}}$ is the squeezed local-oscillator-frequency $\left(\omega_{L O}\right)$ mode parameterized by complex numbers $\alpha$ and $\xi{ }^{13}$ In the target-present case an additional mode is in a nonvacuum state, specifically the coherent state $|\beta\rangle_{k_{T}}$ at the target-return frequency $\omega_{T}$ :

$$
\left|\psi_{1}\right\rangle=|\beta\rangle_{k_{T}}|\alpha, \xi\rangle_{k_{L O}} \prod_{k \neq k_{T}, k_{L O}}|0\rangle_{k}
$$

Using (4), 8)- 11) and the relations 13

$$
\begin{gathered}
\widehat{a}_{k}|0\rangle_{k}={ }_{k}\langle 0| \widehat{a}_{k}^{\dagger}=0, \\
k_{T}\left\langle\beta\left|\widehat{a}_{k_{T}}\right| \beta\right\rangle_{k_{T}}=\beta, \quad{ }_{k_{T}}\left\langle\beta\left|\widehat{a}_{k_{T}}^{\dagger}\right| \beta\right\rangle_{k_{T}}=\beta^{*},
\end{gathered}
$$

and

$$
\begin{aligned}
& k_{k_{L O}}\left\langle\alpha, \xi\left|\widehat{a}_{k_{L O}}\right| \alpha, \xi\right\rangle_{k_{L O}}=\alpha, \\
& k_{k_{L O}}\left\langle\alpha, \xi\left|\widehat{a}_{k_{L O}}^{\dagger}\right| \alpha, \xi\right\rangle_{k_{L O}}=\alpha^{*}
\end{aligned}
$$

we find that

$$
\left\langle\psi_{0}|\widehat{S}| \psi_{0}\right\rangle=0
$$

since the only possible nonzero term, $\widehat{a}_{k_{L O}}^{\dagger} \widehat{a}_{k_{L O}}$, is forbidden by the restriction on the summation in (8), and

$$
\begin{aligned}
\left\langle\psi_{1}|\widehat{S}| \psi_{1}\right\rangle= & \frac{\kappa \hbar}{2 \varepsilon_{0} V}\left(\omega_{T} \omega_{L O}\right)^{1 / 2} \\
& |\alpha||\beta| \cos \left(\theta_{T}-\theta_{L O}+\theta_{H}\right)
\end{aligned}
$$

where

$$
\theta_{T}=\arg \beta, \quad \theta_{L O}=\arg \alpha .
$$

Using (7)-10 and (12),

$$
\begin{gathered}
\left\langle\psi_{0}\left|\widehat{S}^{2}\right| \psi_{0}\right\rangle=\left(\frac{\kappa \hbar}{4 \varepsilon_{0} V}\right)^{2} \\
\sum_{k \text { s.t. }\left|\omega_{L O}-\omega_{k}\right|=\omega_{H}} \quad \text { l s.t. } \mid \sum_{\left|\omega_{l}-\omega_{L O}\right|=\omega_{H}} \omega_{L O}\left(\omega_{k} \omega_{l}\right)^{1 / 2} \\
\left\langle\psi_{0}\left|\widehat{a}_{k_{L O}}^{\dagger} \widehat{a}_{k} \widehat{a}_{l}^{\dagger} \widehat{a}_{k_{L O}}\right| \psi_{0}\right\rangle \\
\exp \left(-i\left[\varepsilon\left(\omega_{L O}-\omega_{k}\right)+\varepsilon\left(\omega_{l}-\omega_{L O}\right)\right] \theta_{H}\right) .
\end{gathered}
$$

Neither $k$ nor $l$ can be equal to $k_{L O}$, due to the restrictions in the summations in 18. If $k \neq l$ then $\widehat{a}_{k}$ and $\widehat{a}_{l}^{\dagger}$ commute, yielding zero since the non- $L O$ modes are in the vacuum state. So the only surviving terms are those for which $k=l$. Using (7),

$$
\left\langle\psi_{0}\left|\widehat{S}^{2}\right| \psi_{0}\right\rangle=\left(\frac{\kappa \hbar}{4 \varepsilon_{0} V}\right)^{2} \sum_{k \text { s.t. }\left|\omega_{L O}-\omega_{k}\right|=\omega_{H}} \omega_{L O} \omega_{k} \bar{n}_{L O}
$$

where

$$
\bar{n}_{L O}={ }_{k_{L O}}\left\langle\alpha, \xi\left|\widehat{a}_{k_{L O}}^{\dagger} \widehat{a}_{k_{L O}}\right| \alpha, \xi\right\rangle_{k_{L O}} .
$$

Using (2), 15) and (19),

$$
\operatorname{Var}_{0} S=\left(\frac{\kappa \hbar}{4 \varepsilon_{0} V}\right)^{2} \sum_{k \text { s.t. }\left|\omega_{L O}-\omega_{k}\right|=\omega_{H}} \omega_{L O} \omega_{k} \bar{n}_{L O} .
$$

The contribution to 21 from the term $\omega_{k}=\omega_{L O}-\omega_{H}$ is termed the "image band" contribution.14

In practice $\omega_{H} \ll \omega_{T}, \omega_{L O}$, so we can take

$$
\omega_{T} \approx \omega_{L O} \equiv \omega
$$

Using (22), (16) and (21) become

$$
\left\langle\psi_{1}|\widehat{S}| \psi_{1}\right\rangle=\frac{\kappa \hbar \omega}{2 \varepsilon_{0} V}|\alpha||\beta| \cos \left(\theta_{T}-\theta_{L O}+\theta_{H}\right),
$$




$$
\operatorname{Var}_{0} S=2\left(\frac{\kappa \hbar \omega}{4 \varepsilon_{0} V}\right)^{2} \bar{n}_{L O} .
$$

Using (15), 23) and (24), the signal-to-noise ratio (1) is

$$
\begin{aligned}
\mathrm{SNR} & =\frac{2|\alpha|^{2}|\beta|^{2} \cos ^{2}\left(\theta_{T}-\theta_{L O}+\theta_{H}\right)}{\bar{n}_{L O}} \\
& =2\left(1-\frac{\sinh ^{2}(r)}{\bar{n}_{L O}}\right) \bar{n}_{T} \cos ^{2}\left(\theta_{T}-\theta_{L O}+\theta_{H}\right)
\end{aligned}
$$

using the relations 13

$$
\begin{gathered}
|\beta|^{2}=\bar{n}_{k_{T}}={ }_{k_{T}}\left\langle\beta\left|\widehat{a}_{k_{T}}^{\dagger} \widehat{a}_{k_{T}}\right| \beta\right\rangle_{k_{T}}, \\
\bar{n}_{L O}=|\alpha|^{2}+\sinh ^{2}(r) .
\end{gathered}
$$

The parameter $r=|\xi|$ is termed the "squeezing parameter." The value $r=0$ corresponds to no squeezing (coherent state). From 25 it is clear that squeezing the LO mode - i.e., letting the LO be in a state with $r>0$ - can only reduce the signal-to-noise ratio.

This result is consistent with the observation by Yuen and Chan, 7 in the context of balanced detection, that while "quantum noise is frequently supposed to arise from local-oscillator (LO) shot noise ... it actually arises from the signal quantum fluctuation." The reasonable but incorrect expectation that squeezing the LO will improve SNR arises from the fact that the variance of the zero-frequency signal, i.e., the time-averaged photoelectric current corresponding to the operator

$$
\widehat{S}^{\prime}=\tau^{-1} \int_{0}^{\tau} d t \widehat{I}(t)=\frac{\kappa \hbar \omega_{k}}{2 \varepsilon_{0} V} \widehat{a}_{k}^{\dagger} \widehat{a}_{k}
$$

(the second equalty holding in the limit $\tau \rightarrow \infty$ ), does change with squeezing. In the target-absent state,

$$
\operatorname{Var}_{0} S^{\prime}=\left\langle\psi_{0}\left|\widehat{S}^{\prime 2}\right| \psi_{0}\right\rangle-\left\langle\psi_{0}\left|\widehat{S}^{\prime}\right| \psi_{0}\right\rangle^{2}
$$

which, for $\tau \rightarrow \infty$ has the value

$$
\operatorname{Var}_{0} S^{\prime}=\left(\frac{\kappa \hbar \omega_{L O}}{2 \varepsilon V}\right)^{2} \operatorname{var} n_{L O}
$$

where

$$
\begin{aligned}
\operatorname{var} n_{L O} & =\left[k_{L O}\left\langle\alpha, \xi\left|\left(\widehat{a}_{k_{L O}}^{\dagger} \widehat{a}_{k_{L O}}\right)^{2}\right| \alpha, \xi\right\rangle_{k_{L O}}\right. \\
& \left.-\left(k_{L O}\left\langle\alpha, \xi\left|\widehat{a}_{k_{L O}}^{\dagger} \widehat{a}_{k_{L O}}\right| \alpha, \xi\right\rangle_{k_{L O}}\right)^{2}\right]
\end{aligned}
$$

For suitable choice of the phase of $\xi$, 31 can indeed be lower than $\bar{n}_{L O}$, the value it takes in a coherent state.13

However, statistical decision theory 10 indicates that if a quantity $S$ computed from measurements made by a detector is used as the decision criterion in a targetdetection task, then it is the variance of that same quantity $S$ that is relevant in evaluating the suitability of $S$ for the task. In heterodyne radar the computed quantitiy $S$ is the Fourier component of the instantaneous photocurrent induced at the detector by the combined target-return and LO beams ${ }^{3}$ The operator corresponding to the instantaneous detector response is $\widehat{I}(t)$, so the operator corresponding to the required Fourier component is $\widehat{S}$ as defined in 3 . It is thus the variance of $\widehat{S}$, not that of $\widehat{S}^{\prime}$, which must be used for computing SNR.

(The general expression for the signal operator (3) for $\tau$ not necessarily infinite, $\omega_{H}$ not necessarily equal to $\left|\omega_{k}-\omega_{l}\right|$ for any $k, l$, is

$$
\begin{gathered}
\widehat{S}=\frac{\kappa \hbar}{2 \varepsilon_{0} V} \sum_{l, k}\left(\omega_{l} \omega_{k}\right)^{1 / 2} \widehat{a}_{l}^{\dagger} \widehat{a}_{k} \exp \left(-i \varepsilon\left(\omega_{l}-\omega_{k}\right) \theta_{H}\right) \\
\cdot \frac{1}{2 i \tau}\left\{\frac{\exp \left(i \theta_{H}\right)}{\omega_{l}-\omega_{k}+\omega_{H}}\left[\exp \left(i\left(\omega_{l}-\omega_{k}+\omega_{H}\right) \tau\right)-1\right]\right. \\
\left.+\frac{\exp \left(-i \theta_{H}\right)}{\omega_{l}-\omega_{k}-\omega_{H}}\left[\exp \left(i\left(\omega_{l}-\omega_{k}-\omega_{H}\right) \tau\right)-1\right]\right\} \cdot(32)
\end{gathered}
$$

This reduces to (8), 28 for the appropriate limiting values of $\tau, \omega_{H}$ and $\theta_{H}$.)

M. A. R. thanks Jonathan Ashcom and Jae Kyung for a helpful discussion on mixing efficiency.

\section{References}

1. D. F. Walls and G. J. Milburn, Quantum Optics (Springer, Berlin, 1994).

2. C. M. Caves, Phys. Rev. D 23, 1693 (1981).

3. R. H. Kingston, Optical Sources, Detectors and Systems: Fundamentals and Applications, (Academic Press, San Diego, 1995).

4. Y.-q. Li, D. Guzun, and M. Xiao, Phys. Rev. Lett. 82, 5225 (1999).

5. T. C. Ralph, Phys. Rev. Lett. 85, 677 (2000).

6. G. Hernandez, Fabry-Perot Interferometers (Cambridge, 1986).

7. H. P. Yuen and and V. W. S. Chan, Opt. Lett. 8, 177 (1983).

8. V. Annovazzi-Lodi, S. Donati and S. Merlo, Opt. Quan. Electron. 24, 258 (1992).

9. Y.-q. Li, P. Lynam, M. Xiao, and P. J. Edwards, Phys. Rev. Lett. 78, 3105 (1997).

10. C. W. Helstrom, Quantum Detection and Estimation Theory (Academic, New York, 1976).

11. K. Gottfreid and T.-M. Yan, Quantum Mechanics: Fundamentals, 2nd. ed. (Springer, New York, 2003).

12. R. J. Glauber, "Optical coherence and photon statistics," in Quantum Optics and Electronics, C. DeWitt, A. Blandin, and C. Cohen-Tannoudji, eds. (Gordon and Breach, New York, 1965).

13. C. G. Gerry and P. L. Knight, Introductory Quantum Optics (Cambridge, 2005).

14. H. A. Haus, Electromagnetic Noise and Quantum Optical Measurements (Springer, Berlin, 2000). 